\title{
A new local approach model for cleavage fracture in ferritic steels and its validation
}

\author{
Guian Qian ${ }^{1, *}$, Wei-Sheng Lei ${ }^{2, *}$, Markus Niffenegger ${ }^{1}$ \\ ${ }^{1}$ Paul Scherrer Institute, Nuclear Energy and Safety Department, Laboratory for Nuclear Materials, OHSA/06, 5232 Villigen PSI, \\ Switzerland \\ ${ }^{2}$ Applied Materials, Inc., 974 East Arques Avenue, Sunnyvale, CA 94085, USA
}

\begin{abstract}
The paper discusses and applies a statistical approach to correlate the fracture behavior of a notched and fracture mechanics specimen. The method can be used for fatigue analysis. The random nature of cleavage fracture process determines that both the microscopic fracture stress and the macroscopic properties including fracture load, fracture toughness and the ductile to brittle transition temperature are all stochastic parameters. This understanding leads to the proposal of statistical assessment of cleavage induced notch toughness of ferritic steels according to a new local approach to cleavage fracture. The temperature independence of the two Weibull parameters in the new model induces a master curve to correlate the fracture load at different temperatures. A normalized stress combining the two Weibull parameters and the yield stress is proposed as the deterministic index to measure notch toughness. This proposed index is applied to compare the notch toughness of a ferritic steel with two different microstructures.
\end{abstract}

\section{Introduction}

Ferritic steels are commonly used to fabricate reactor pressure vessels (RPVs) in the commercial light water reactors. Ferritic steels have body centered cubic crystal structures that possess the ductile-to-brittle transition temperature (DBTT) characteristic $[\mathbf{1}, \mathbf{2}]$. This leads to the inherent susceptibility of RPVs to stochastic transgranular cleavage induced brittle fracture in the actual service temperature range due to geometrical discontinuities at notches and cracks, and in the occurrence of pressurized thermal shock loadings. Both deterministic and statistical approaches have been developed to evaluate structural integrity of RPVs against brittle fracture. As reviewed in [3], the conventional transition temperature method, fracture mechanics, and fracture physics approaches are deterministic; the Master Curve (MC) method [4] and most of the Local Approach (LA) [5] are statistical. The MC method empirically describes cleavage fracture toughness distribution using a three-parameter Weibull distribution with fixed values of the modulus $(\mathrm{m}=4)$ and the threshold toughness $\left(K_{\min }=20 M P a \sqrt{m}\right)$, with only the scale parameter $\left(\mathrm{K}_{0}\right)$ to be determined by fitting fracture toughness $\left(\mathrm{K}_{\mathrm{Ic}}\right)$ data [4]. LA aims to develop a predictive, rather than descriptive, statistical model inspired by a pertinent physical understanding of cleavage process. The Beremin model [5] was the pioneering work in LA for cleavage fracture. The Beremin model is essentially a two-parameter Weibull distribution as below:

$$
P=1-\exp \left[-\left(\sigma_{W} / \sigma_{0}\right)^{m}\right]
$$

with $\sigma_{W}=\left(\int_{V_{p}} \sigma_{1}^{m} \cdot d V / V_{0}\right)^{1 / m}$

where $\mathrm{P}$ is the cumulative probability of failure, $\mathrm{V}_{\mathrm{p}}$ denotes the volume of the plastic deformation zone as the cleavage fracture process zone, $\mathrm{m}$ and $\sigma_{0}$ are the two model parameters known as Weibull modulus and the scale parameter, respectively, $\sigma_{1}$ is the maximum tensile principal stress, $\mathrm{V}_{0}$ is an elementary volume representing the mean volume occupied by each micro-crack in a solid, $\mathrm{dV}$ is the differential volume, $\sigma_{W}$ denotes the Weibull stress.

The Beremin model has suffered from the ambiguity in model parameter calibration, i.e., the variation of the model parameters with temperature and geometrical constraint [6-10].

Recent studies [3, 13, 14] have identified the fundamental defects of the Beremin model, which are summarized in Table 1. Note that some of the highlighted fundamental defects are inherited by those modifications to the Beremin model, such as the incompliance with the physical assumption of plastic yielding as a prerequisite to cleavage fracture due to the adoption of a fixed-value threshold stress $\sigma_{\text {th }}$ (including the case of $\sigma_{\text {th }}=0$ ) [6-10], and the violation to the normality axiom of probability owing to the adoption of Eq. (6a) as the basic formulation of cumulative probability P [10]. An example was elaborated in detail in the commentary [15] to the work in [10]. In Table 1, the necessary corrections to the Beremin model are also 
provided to ensure the mathematical rigorousness and the physical compliance with the five assumptions below:

(1).The uniform spatial distribution of microcracks

(2). The weakest-link postulate of brittle fracture

(3).Plastic yielding as a prerequisite for cleavage fracture

(4). The maximum tensile principal stress criterion for cleavage fracture (Eq.(8))

(5).The power-law distribution of microcrack size (Eq.(9))

Basically, equations are defined in Table 1 to translate the five assumptions into mathematical formulations and check the validity of each step for formulating the Beremin model. As a result of these necessary corrections, the Beremin model in Eq. (1) or Eq.(8a) is reformulated to Eq.(8b) in strict conformity to the five assumptions.

Accordingly, by adopting a three-parameter Weibull distribution density function of microscopic cleavage fracture stress $S$ in place of the power law distribution of microcrack size (a) (Eq.(9) in Table 1) as assumption (5),

$$
\begin{aligned}
& g(S)= \\
& m \cdot\left[\left(S-\sigma_{t h}\right)^{m-1} / \sigma_{0}^{m}\right] \cdot \exp \left[-\left(S-\sigma_{t h}\right)^{m} / \sigma_{0}^{m}\right]
\end{aligned}
$$

\begin{tabular}{|c|c|c|c|}
\hline \multicolumn{3}{|c|}{ Beremin model and corrections } & \multirow{2}{*}{$\begin{array}{l}\text { Equation / } \\
\text { inequation } \\
\text { Number }\end{array}$} \\
\hline & Beremin Model & Corrections to Beremin Model & \\
\hline Model assumption \#1 & \multicolumn{2}{|l|}{ Uniform spatial distribution of microcracks } & \\
\hline Model assumption \#2 & \multicolumn{2}{|l|}{ Weakest-link model } & \\
\hline $\begin{array}{l}\text { Basic formulation of } \\
\text { cumulative probability } \\
P(V)\end{array}$ & $P(V)=1-\exp \left[-\int_{V} p\left(\sigma, V_{0}\right) \frac{\delta V}{V_{0}}\right]$ & $P(V)=1-\exp \left\{\int_{V} \ln \left[1-p\left(\sigma, V_{0}\right)\right] \frac{\delta V}{V_{0}}\right\}$ & $(3 a, b)$ \\
\hline Self-consistency check & $\begin{array}{l}V=V_{0}: \\
\text { Eq. }(1 \mathrm{a}) \Rightarrow P\left(V_{0}\right)=1-\exp \left[-p\left(\sigma, V_{0}\right)\right] \\
\text { False! }\end{array}$ & $\begin{array}{l}V=V_{0}: \\
\text { Eq. }(1 \mathrm{~b}) \Rightarrow P\left(V_{0}\right)=p\left(\sigma, V_{0}\right) \\
\text { True! }\end{array}$ & $(4 a, b)$ \\
\hline Model assumption \#3 & \multicolumn{2}{|c|}{$\begin{array}{l}\text { Plastic yielding as a prerequisite for cleavage fracture } \\
\Rightarrow \text { Initial yielding as the threshold stress state for cleavage fracture. }\end{array}$} & \\
\hline Model assumption \#4 & \multicolumn{2}{|l|}{ Microscopic fracture criterion : $\sigma=\sigma_{1} \geq S$} & (5) \\
\hline Model assumption \#5 & \multicolumn{2}{|c|}{ Power-law distribution of microcrack size: $f(a)=k a^{-v}(k>0, v>1), m=2 v-2$} & (6) \\
\hline $\begin{array}{l}\text { Fracture probability of a } \\
\text { volume element } V_{0}, p\left(\sigma, V_{0}\right)\end{array}$ & $p\left(\sigma, V_{0}\right)=\int_{a\left(\sigma_{1}\right)}^{\infty} f(a) d a=\left(\frac{\sigma_{1}}{\sigma_{0}}\right)^{m}$ & $\begin{array}{r}p\left(\sigma, V_{0}\right)=\int_{a\left(\sigma_{1}\right)}^{a_{\max }\left(\sigma_{t h}=\sigma_{1,0}\right)} f(a) d a \\
=\frac{\sigma_{1}^{m}-\sigma_{1,0}^{m}}{\sigma_{0}^{m}-\sigma_{1,0}^{m}}\end{array}$ & $(7 \mathrm{a}, \mathrm{b})$ \\
\hline Cumulative probability & $P(V)=1-\exp \left[-\int_{V}\left(\frac{\sigma_{1}}{\sigma_{0}}\right)^{m} \frac{\partial V}{V_{0}}\right]$ & $P(V)=1-\exp \left[\int_{V} \operatorname{Ln}\left(\frac{\sigma_{0}^{m}-\sigma_{1}^{m}}{\sigma_{0}^{m}-\sigma_{1,0}^{m}}\right) \frac{\delta V}{V_{0}}\right]$ & $(8 \mathrm{a}, \mathrm{b})$ \\
\hline Model applicable range & $\begin{array}{l}0 \neq\left(\frac{\sigma_{y s}}{\sigma_{0}}\right)^{m} \leq p\left(\sigma, V_{0}\right) \leq 1 \Rightarrow \sigma_{y s} \leq \sigma_{1} \leq \\
\sigma_{0} \\
\text { Violate normality axiom of probability. }\end{array}$ & $0 \leq p\left(\sigma, V_{0}\right) \leq 1 \Rightarrow \sigma_{1,0} \leq \sigma_{1} \leq \sigma_{0}$ & $(9 a, b)$ \\
\hline $\begin{array}{l}\text { Assessment of cumulative } \\
\text { probability }\end{array}$ & $\begin{array}{c}\quad 0 \leq P(V) \leq 1-\exp \left(-V / V_{0}\right) \neq 1 \\
\text { Violate normality axiom of probability }\end{array}$ & $0 \leq P(V) \leq 1$ & $(10 \mathrm{a}, \mathrm{b})$ \\
\hline Key references & {$[5,10]$} & {$[3,13,14]$} & \\
\hline Notes & $\begin{array}{l}\text { a - microcrack size; } \mathrm{S}-\text { strength of } \mathrm{vc} \\
\text { plastic deformation zone as fracture } \mathrm{p} \\
\text { effective stress to induce cleavage fract } \\
\left(\mathrm{S} \geq \sigma_{\mathrm{th}}\right) ; \sigma_{1,0^{-}} \text {maximum tensile princip } \\
\sigma_{\mathrm{ys}} \text {-yield stress; } \mathrm{k}, \mathrm{v}, \mathrm{m}, \sigma_{0} \text {-model paran }\end{array}$ & $\begin{array}{l}\text { ne } V_{0} \text { with an embedded microcrack of } s \\
\text { ess zone; } V_{0^{-}} \text {average volume occupied } \\
\sigma_{1^{-}} \text {maximum tensile principal stress; } \sigma_{\text {th }} \\
\text { stress of a differential volume element ( } \delta \\
\text { ers as material constants. }\end{array}$ & $\begin{array}{l}\text { a; V- volume of } \\
\text { a microcrack; } \sigma \text { - } \\
\text { threshold strength }\end{array}$ \\
\hline
\end{tabular}

Table 1. Comparison of Beremin model and its corrections. 
a new local approach to cleavage fracture was proposed $[3,13]$ :

$$
p\left(\sigma, V_{0}\right)=\int_{\sigma_{t h}=\sigma_{1,0}}^{\infty} g(S) d S=1-\exp \left(\frac{\sigma_{1}-\sigma_{1,0}}{\sigma_{0}}\right)^{m}
$$

$P=1-\exp \left\{\int_{V_{p}} \ln \left[1-p\left(\sigma, V_{0}\right)\right] \mathrm{dV} / V_{0}\right\}=1-$ $\exp \left[-\left(\sigma_{W} / \sigma_{0}\right)^{m}\right]$

$\sigma_{W}=\left[\int_{V_{p}}\left(\sigma_{1}-\sigma_{1,0}\right)^{m} \cdot d V / V_{0}\right]^{1 / m}$

where $\sigma_{\mathrm{W}}$ is the Weibull stress. Note that the essential difference between Eq.(14) and Eq. (3) lies in that $\sigma_{1,0}$ in Eq.(14) varies with temperature, strain rate and stress state, while $\sigma_{t h}$ in Eq.(3) is a fixed-value independent of temperature, strain rate and stress state.

The present work tries to explore this aspect by applying the new local approach model in Eq. (12) to assess the published datasets for notch toughness that was already analyzed by deterministic approaches. The focus is to statistically assess and compare the notch toughness. Further, both the deterministic and the statistical approaches will be used to analyze the notch toughness.

\section{Experimental data and method of calibration}

\subsection{Experimental data}

The experimental data of a rolled C-Mn pressure vessel steel $16 \mathrm{MnR}$ reported by Wang et al. in [18] are used. Cleavage initiation in notched specimens with carbides and inclusions was investigated at $77 \mathrm{~K}$ and $143 \mathrm{~K}$. The same steel went through different heat treatments to obtain the same ferrite grains but with fine carbide (FC) and coarse carbide (CC) particles, respectively. The mechanical tests, scanning electron microscopy (SEM) analysis and measurements, and finite element analysis (FEA) were employed to compare the notch toughness of the specimens with FC and CC particles.

The static four point bending (4PB) tests were conducted at $77 \mathrm{~K}$ and $143 \mathrm{~K}$ on $45^{\circ}$-angle $\mathrm{V}$-notched prismatic beams in Fig.1a. The general yield load $\mathrm{P}_{\text {gy }}$ of the 4PB specimens was calculated by $P_{g y}=0.7045 \sigma_{y s} B(W-a)^{2} / L$, where B is specimen thickness, $\mathrm{W}$ the height, $\mathrm{a}=4.25 \mathrm{~mm}$ the notch depth, and $\mathrm{L}$ the bending span, with $\mathrm{L}=\mathrm{B}=\mathrm{W}=12.7 \mathrm{~mm}$. Fig.2 summarizes the measured fracture loads $\mathrm{P}_{\mathrm{f}}$.

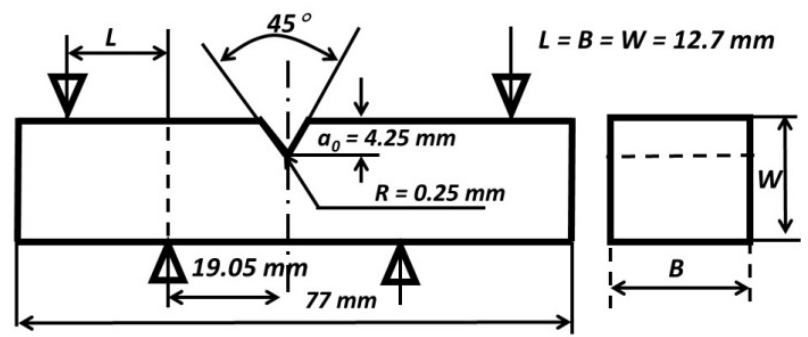

Fig. 1a. Four-point bending specimen: geometry [18].

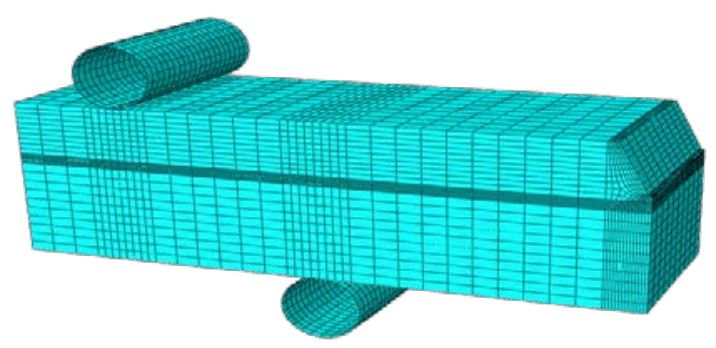

Fig. 1b. Four-point bending specimen: finite element model.

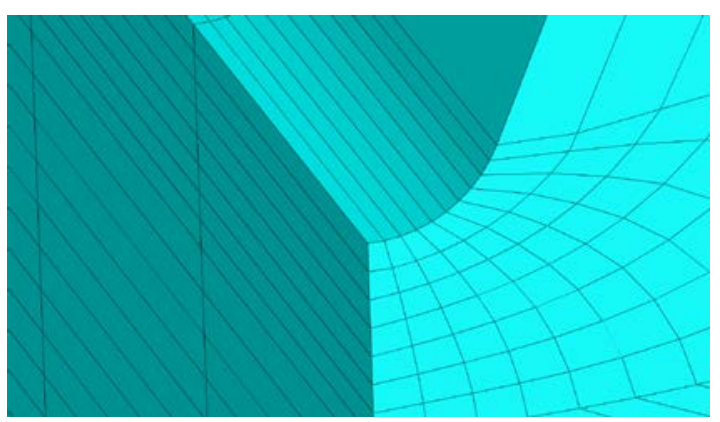

Fig. 1c. Four-point bending specimen: layout of finite elements at notch tip.

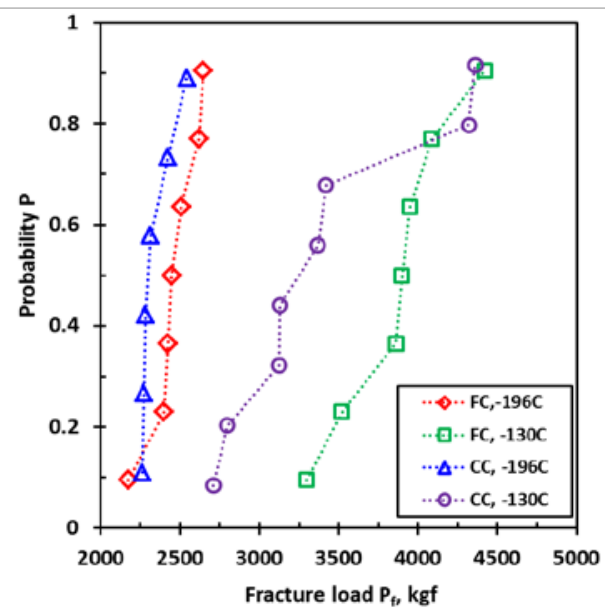

Fig. 2. Experimental results of fracture loads $P_{f}[\mathbf{1 8}]$. 


\subsection{Method of calibration}

In the present study, finite element analysis (FEA) is conducted using ABAQUS software version 6.14 for stress distribution in the 4PB specimen. 20-node brick elements are used. Only a quarter of the specimen was modeled due to symmetry with a total of 15940 elements as shown in Fig.1b and Fig.1c.

Eq. (13) is rewritten as

$$
\operatorname{Ln} \operatorname{Ln}[1 /(1-P)]=m \operatorname{Ln}\left(\sigma_{W}\right)-m \operatorname{Ln}\left(\sigma_{0}\right)
$$

The calibration method for Eq. (15) was developed in [19]. Fig.3 shows the flow chart of calibration. Here we just briefly highlight a few key points for the calibration. In addition to a subroutine being written to extract the stress and strain distributions for different elements to calculate the newly defined Weibull stress $\sigma_{W}$ in Eq. (14), the key challenge is to properly calculate the threshold stress $\sigma_{1,0}$ for the initial yielding of a finite volume element $\Delta V$ to enable the calculation of $\sigma_{\mathrm{W}}$. As elaborated in the Introduction section, due to the nonuniform stress distributions in a notched specimen, the moment for each volume element to start initial plastic yielding and the amplitude of the stress $\sigma_{1,0}$ at the initial yielding therein are different. In theory, we can determine the stress $\sigma_{1,0}$ in each finite volume element $\Delta \mathrm{V}$ by FEA with the experimentally measured true elastic-plastic hardening stress-strain curve of the steel. Since during the loading process, the stress state in each volume element keeps changing. At each loading step, while some elastic volume elements reach the initial plastic yielding state, the other already plastically yielded volume elements in the preceding loading step will go through strain hardening phase. This demands a sufficiently small loading step in order to precisely capture the moment of initial plastic yielding state of each volume element. This would translate into a significant amount of numerical calculations to determine the values of $\sigma_{1,0}$ in all relevant volume elements. Therefore, this path is not practically desirable. An alternative and cost-effective approach to determine the values of $\sigma_{1,0}$ in different volume elements has been thus developed in [19]. In essence, it adopts an elasticperfectly plastic material model for FEA to determine the value of $\sigma_{1,0}$ in each volume element. The resultant value of stress $\sigma_{1}$ in a plastically deformed volume element is stored as $\sigma_{1,0}$ for that particular volume element. Then the experimentally measured true elasticplastic hardening stress-strain curve of the steel in Table 1 is used for FEA to calculate the stress $\sigma_{1}$ in each volume element at a given load $\mathrm{P}_{\mathrm{f}}$. Next, substituting the values of the instant stress $\sigma_{1}$ and the threshold stress $\sigma_{1,0}$ for exactly the same volume element one by one into Eq. (14) to calculate $\sigma_{\mathrm{W}}$ at each load as input to Eq. (15). Then the same conventional calibration procedure for Eq. (15) in [19] is applied to obtain the values of $m$ and $\sigma_{0}$. The proposed approach to determining the values of $\sigma_{1,0}$ in different volume elements according to an elasticperfectly plastic material model significantly reduces the amount of numerical calculation. The stress redistribution between a perfectly plastic volume element and an elastic volume can be different from that between a plastic hardening volume element and an elastic volume. However, since we adopt the same values of elastic modulus, Poisson's ratio and yield stress for the real material in the elastic-perfectly plastic material model, we expect the difference in the values of $\sigma_{1,0}$ determined using an elastic-perfectly plastic material model and using a true elastic-plastic hardening material model is insignificant. The reader is reminded that in contrast to the conventional fixed-value threshold, the proposed approach to the determination of $\sigma_{1,0}$ pertinently captures the most critical characteristics of threshold stress, that is, the temperature and stress state dependence of threshold. In our future work, we expect to conduct a direct comparison of the values of $\sigma_{1,0}$ determined using the afore cited two different material models.

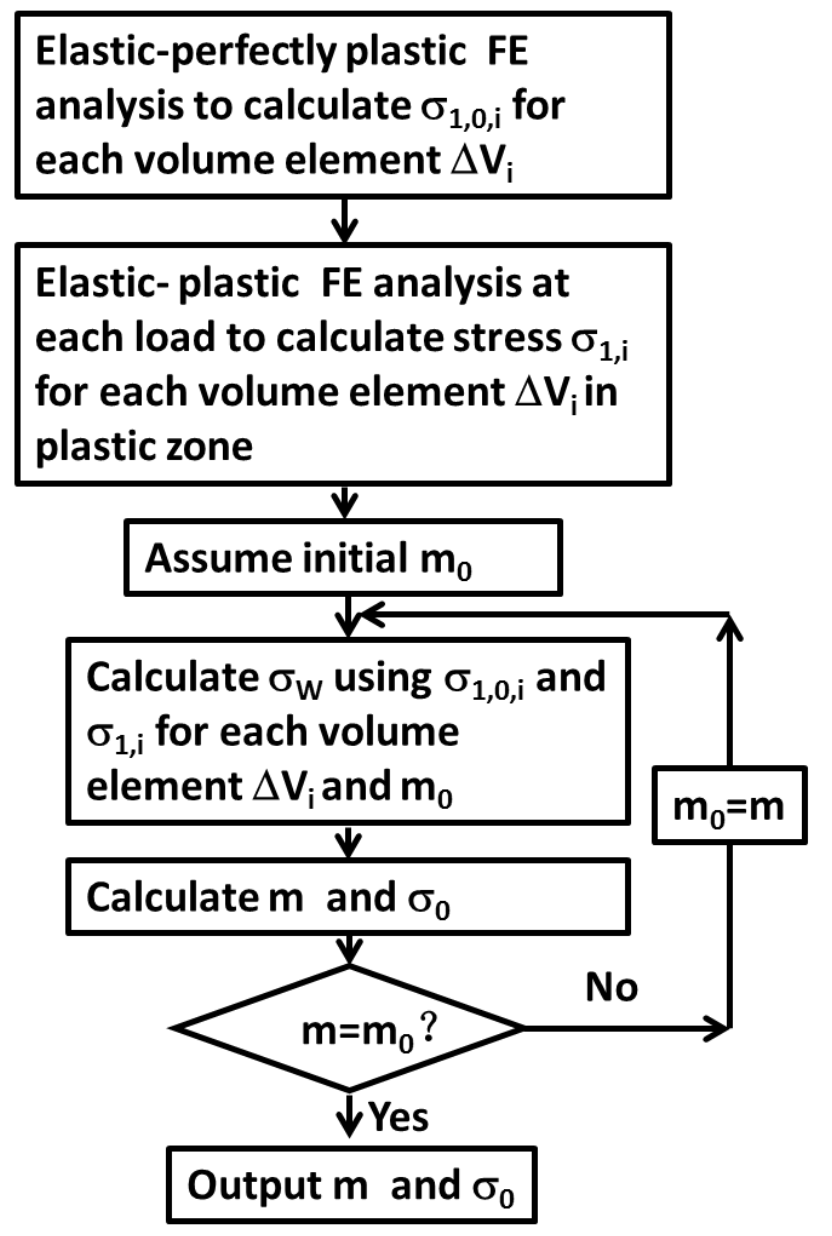

Fig. 3. Flow chart for the calibration of Eq.(15).

\section{Results}

Using the experimental data in Fig.2 as input, Eq.(15) was calibrated at $77 \mathrm{~K}$ for $\mathrm{FC}$ and $\mathrm{CC}$ specimens, respectively. Fig. 4 shows the calibration results at $77 \mathrm{~K}$. For $\mathrm{FC}$ specimens, $\mathrm{m}=25.6, \sigma_{0}=228.3 \mathrm{MPa}$; For $\mathrm{CC}$ specimens, $\quad \mathrm{m}=11, \sigma_{0}=161.1 \mathrm{MPa}$. With the 
calibrated parameters, Weibull stress at different loadings are calculated for the temperature of $77 \mathrm{~K}$ and 143K, as shown in Fig. 5a and $\mathbf{5 b}$.

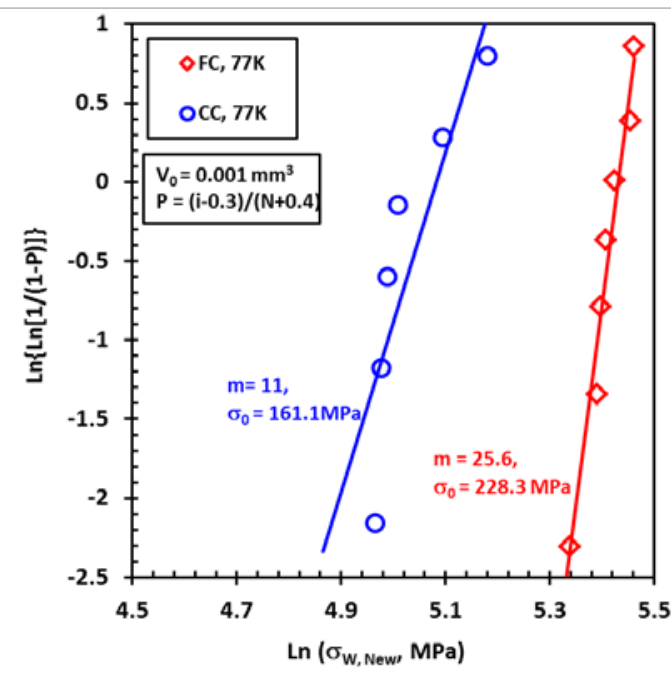

Fig. 4. Summary of calibration results of Eq.(18) at 77K.

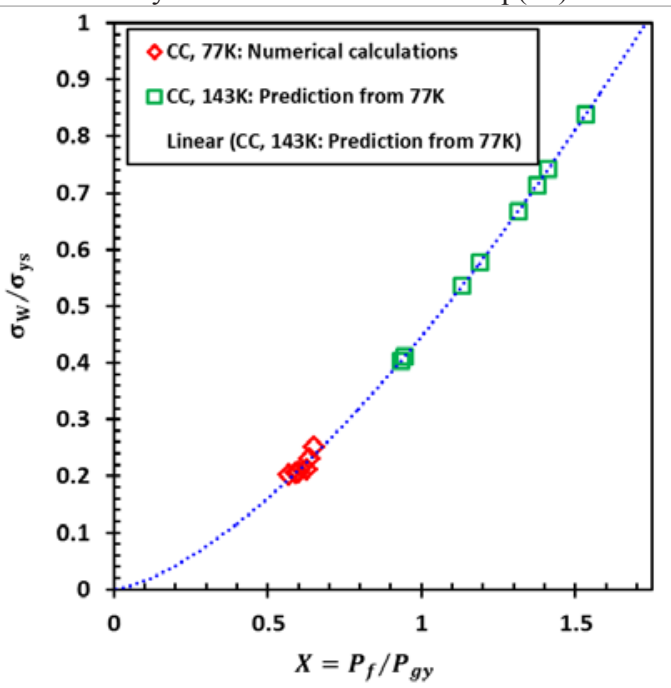

Fig. 5a. Weibull stress at $77 \mathrm{~K}$ and $143 \mathrm{~K}$ for the $\mathrm{CC}$ material.

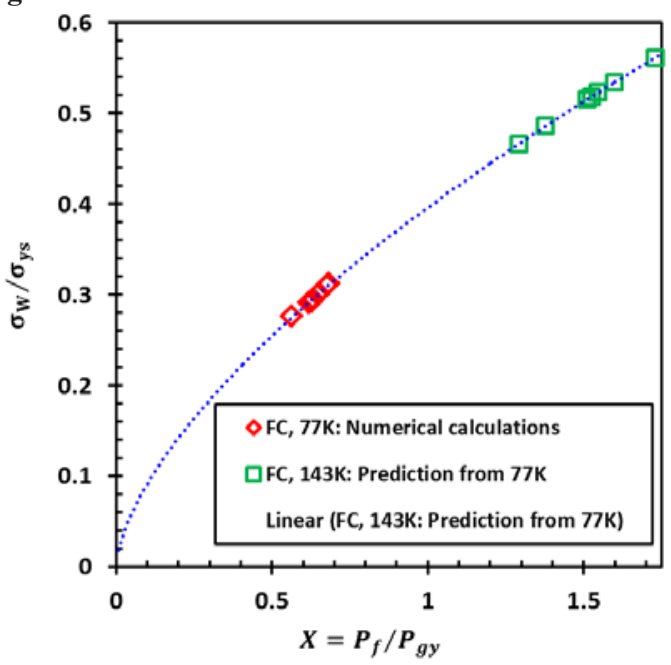

Fig. 5b. Weibull stress at $77 \mathrm{~K}$ and $143 \mathrm{~K}$ for the FC material.
The new model Eq.(13) assumes temperature independence of model parameters, so we did not calibrate Eq. (15) at $143 \mathrm{~K}$, but will justify this in the following. As discussed in detail in [19], according to the definition of the new Weibull stress $\sigma_{W}$ in Eq.(14), if the two model parameters ( $\mathrm{m}$ and $\sigma_{0}$ ) are indeed intrinsic material properties independent of temperature, for a given material and a fixed specimen geometry, $\sigma_{W}$ is a function of the fracture load $P_{f}$ at a fixed temperature $(\mathrm{T})$ :

$\sigma_{W}(T)=F\left(P_{f}(T)\right)$

Alternatively, Eq. (16) can be converted to the following format that allows considering temperature effect:

$\frac{\sigma_{W}(T)}{\sigma_{y s}(T)}=F\left(\frac{P_{f}(T)}{P_{g y}(T)}\right)$

or $\quad \sigma_{W}(T)=\sigma_{y s}(T) \cdot F\left(\frac{P_{f}(T)}{P_{g y}(T)}\right)$

where the general yield load $\mathrm{P}_{\mathrm{gy}}$ is defined in Section 2.1 Experimental data.

In order to validate the temperature independence of Weibull modulus m, we now substitute Eq. (17b) in Eq. (13) to get

$P=1-\exp \left[-\left(\frac{\sigma_{y s}}{\sigma_{0}}\right)^{m} F^{m}\left(\frac{P_{f}(T)}{P_{g y}(T)}\right)\right]$

Eq. (18) is further rewritten as,

$Y=\frac{1}{\sigma_{y s}} \cdot\left\{\ln \left[\frac{1}{(1-P)}\right]\right\}^{\frac{1}{m}}=\frac{1}{\sigma_{0}} \cdot F\left(\frac{P_{f}(T)}{P_{g y}(T)}\right)$

A master curve behavior between the compound parameters $Y=\frac{1}{\sigma_{y s}} \cdot\left\{\ln \left[\frac{1}{(1-P)}\right]\right\}^{\frac{1}{m}}$ and $X=\frac{P_{f}(T)}{P_{g y}(T)}$ is thus expected regardless of the test temperature, only if the temperature independence of two model parameters (m and $\sigma_{0}$ ) holds. According to Eq. (19), the experimental data in Fig.2 at two different temperatures are transferred into Fig.6a and $\mathbf{b}$ for the expected correlation with the calibrated values of $m=25.4$ for $\mathrm{FC}$ and $m=11$ for $\mathrm{CC}$ in sequence.

As shown in Fig.6a and b, the experimental results at both temperatures fall onto a single "master curve" that correlates the compound parameters $Y=\frac{1}{\sigma_{y s}}$. $\left\{\ln \left[\frac{1}{(1-P)}\right]\right\}^{\frac{1}{m}}$ and $X=\frac{P_{f}(T)}{P_{g y}(T)}$ for each material. What underpins the "master curve" behavior is the temperature independence of Weibull parameters $\mathrm{m}$ and $\sigma_{0}$. Therefore, Fig. $\mathbf{6}$ a and $\mathbf{b}$ validate the assumption of Eq. (13) on the temperature independence of model parameters $\mathrm{m}$ and $\sigma_{0}$. 


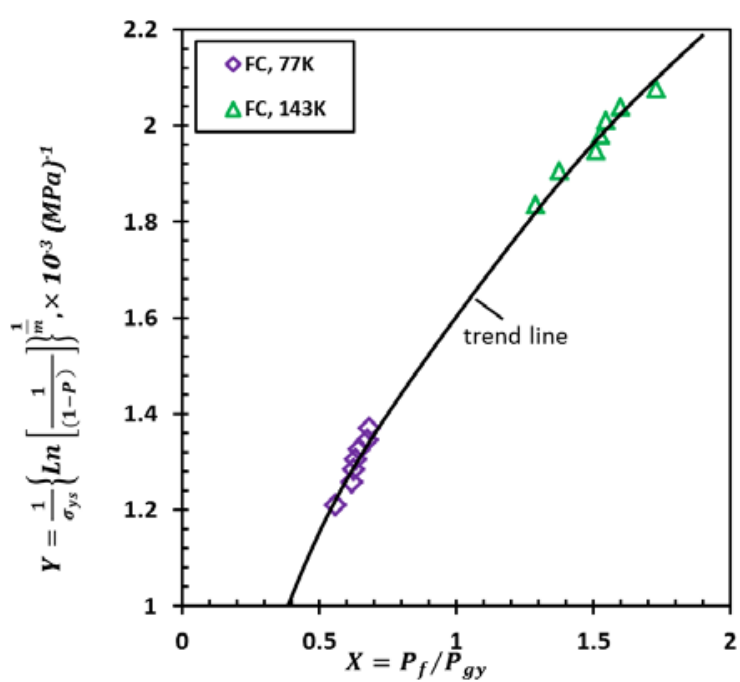

Fig. 6a. Correlation between the compound parameters $Y=\frac{1}{\sigma_{y s}} \cdot\left\{\ln \left[\frac{1}{(1-P)}\right]\right\}^{\frac{1}{m}}$ and $X=\frac{P_{f}(T)}{P_{g y}(T)}$ at two different temperatures for specimens FC.

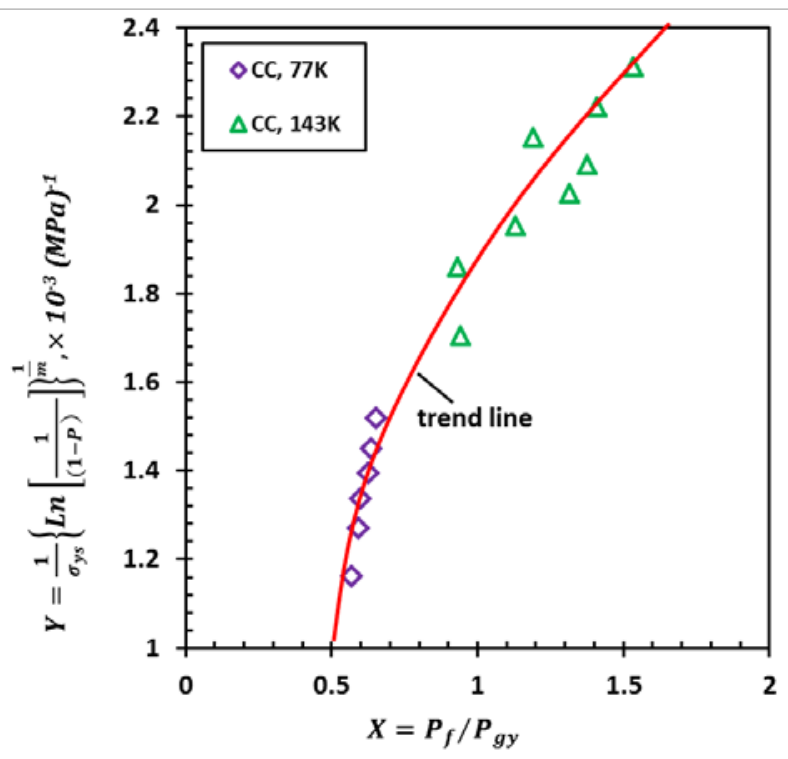

Fig. 6b. Correlation between the compound parameters $Y=\frac{1}{\sigma_{y s}} \cdot\left\{\ln \left[\frac{1}{(1-P)}\right]\right\}^{\frac{1}{m}}$ and $X=\frac{P_{f}(T)}{P_{g y}(T)}$ at two different temperatures for specimens CC.

\section{Conclusions}

As an effort to adopt a fracture physics based statistical approach for correlating fracture properties of a simple and less expensive notched specimen and a costly fracture mechanics specimen, a new statistical model of cleavage fracture is discussed and applied to assess notch toughness of ferritic steels against cleavage fracture by analyzing published cleavage fracture datasets of $16 \mathrm{Mn}$ steel with fine and coarse carbides (FC and CC) and at two test temperatures in [18]. The following conclusions can be drawn:
A new local approach to fracture and the calibration method are discussed. The proposed calibration method for a new local approach to cleavage fracture is proved feasible using experimental data.

The proposed calibration method for a new local approach to cleavage fracture is proved feasible using experimental data.

There exists a master curve to correlate the compound parameters $Y=\frac{1}{\sigma_{y s}} \cdot\left\{\ln \left[\frac{1}{(1-P)}\right]\right\}^{\frac{1}{m}}$ and $X=\frac{P_{f}(T)}{P_{g y}(T)}$ at two different temperatures for each microstructure (FC and $\mathrm{CC})$. This validates the temperature independence of two model parameters ( $\mathrm{m}$ and $\sigma_{0}$ ).

It should be noted that the weakest link based approach can be used for the fatigue analysis, especially for the very-high-cycle fatigue analysis where crack initiation from the inhomogeneity inside the material occurs.

\section{References}

1. Zhang, Z., and Qian, X. (2017) Fatigue Fract. Eng. Mater. Struct., 40, 1128-1142.

2. Chao, Y.J., Yang, S., and Sutton, M.A. (1994) J. Mech. Phys. Solids, 42, 629-647.

3. Lei, W.-S. (2016) Philos. Mag., 96, 3586-3631.

4. Wallin, K. (2002) Eng. Fract. Mech., 69, 451-481.

5. Pineau, A. (2006) Int. J. Fract., 138, 139-166.

6. Bakker, A., and Koers, R.W.J. (1991) ESIS/EGF9, J.G. Blauel and K.H. Schwalbe, eds., Mechanical Engineering Publications, London, pp. 613-632.

7. Hausild, P., Berdin, C., and Bompard, P. (2005) Mater. Sci. Eng. A, 391, 188-197.

8. Moattari, M., Sattari-Far, I., Persechino, I., and Bonora, N. (2016) Mater. Sci. Eng. A, 657, 161172.

9. Petti, J.P. and Dodds, R.H. (2005) Eng. Fract. Mech., 72, 91-120.

10. Ruggieri, C., Savioli, R.G., Dodds, R.H. (2015) Eng. Fract. Mech., 146, 185-209.

11. Heerens, J., Pfuff, M., Hellmann, D., and Zerbst, U. (2002) Eng. Fract. Mech., 69 , 483-495.

12. Lei, W.-S. (2016) Mech. Mater., 101, 81-92.

13. Lei, W.-S. (2016) Mech. Mater., 93, 184-198.

14. Lei, W.-S. (2016) Philos. Mag. Lett., 96, 101-111.

15. Lei, W.-S. (2017) Eng. Fract. Mech., 178, 527-534.

16. Lei, W.S., Li, D.M., Yao, M. (1996) Mater. Chem.Phys., 46, 1-5.

17. Kotrechko, S., Gryschchenko, V., Kozak, V., Dlouhy, I. (2016) Solid State Phenomena, 258, 281285

18. Wang, G. Z., Liu, Y. G., Chen, J. H. (2004) Mater. Sci. Eng. A, 369, 181-191.

19. Qian, G., Lei, W.-S. , Niffenegger, M. (2017) Mater. Sci. Eng. A, 694, 10-12. 